\title{
Un exemple de mémoire " disputée » : les deux monuments aux morts de Ploujean
}

\section{Patrick Gourlay}

\section{(2) OpenEdition}

\section{Journals}

\section{Édition électronique}

URL : http://journals.openedition.org/abpo/1631

DOI : 10.4000/abpo. 1631

ISBN : 978-2-7535-1485-0

ISSN : 2108-6443

Éditeur

Presses universitaires de Rennes

Édition imprimée

Date de publication : 20 mars 2002

Pagination : 129-145

ISBN : 978-2-86847-708-8

ISSN : 0399-0826

\section{Référence électronique}

Patrick Gourlay, « Un exemple de mémoire « disputée » : les deux monuments aux morts de

Ploujean », Annales de Bretagne et des Pays de l'Ouest [En ligne], 109-1 | 2002, mis en ligne le 20 mars 2004, consulté le 20 avril 2019. URL : http://journals.openedition.org/abpo/1631 ; DOI : 10.4000/ abpo.1631 


\title{
Un exemple de mémoire " disputée ": les deux monuments aux morts de Ploujean
}

\author{
Patrick GOURLAY \\ Professeur d'histoire-géographie au collège de Lanmeur \\ " Je reste troublé par le spectacle que donnent \\ le trop de mémoire ici, le trop d'oubli ailleurs. \\ Une politique de la juste mémoire \\ est un de mes thèmes civiques avoués." \\ (Paul Ricœur, La Mémoire, l'Histoire, l'Oubli, \\ Le Seuil, décembre 2000.)
}

Marc Bloch l'a souligné dans son Apologie pour l'histoire : "L'historien doit très consciemment prendre son point de départ dans le présent. " Aujourd'hui, à Ploujean (Finistère), la Grande Guerre entraîne encore deux types de cérémonies du souvenir. La première, officielle et lä̈que, est nationale : elle se déroule comme partout en France, chaque 11 novembre et honore les disparus de la Première Guerre mondiale. La seconde, privée et religieuse, est plus discrète et se tient dans la chapelle de l'ancienne propriété de Ferdinand Foch. Depuis la mort au front, le même jour d'août 1914, de son fils et de son gendre, chaque 22 août, une messe est dite en hommage à Germain Foch et à Paul Bécourt. Cette cérémonie se célèbre encore de nos jours en présence de l'arrière petite-fille du maréchal ${ }^{1}$.

Si ces deux cérémonies ne sont évidemment ni rivales, ni de même dimension, elles sonnent toutefois comme un symbole de la mémoire " disputée " de la Grande Guerre à Ploujean : ici, le souvenir du conflit ne se conjugua pas avec l'union sacrée. Il fut nourri d'oppositions et de divisions; on marqua sa différence, on affirma son camp : laïc ou clérical, républicain contre monarchiste. Cette opposition s'affirme par la présence de deux monuments aux morts : l'un civique et laïc; l'autre religieux. Il est vrai qu'en Bretagne, à côté du monument commémoratif installé sur la place publique,

1. Entretien avec Sœur Marie Emmanuelle, des Petites Sœurs de Saint François, le 2 juin 2001. Il s'agit de la Mère supérieure de la congrégation religieuse installée, depuis le début des années 1980, dans l'ancienne propriété du maréchal Foch. 
on trouve, assez souvent, une plaque dans l'église ou sous le porche. Mais ce qui fait l'originalité de cet exemple ploujeannais, c'est l'impossibilité à accepter un monument fédérateur. L'idée de compromis, en cette période d'union sacrée, est ici inconcevable à cause de l'antagonisme toujours présent entre héritiers des Blancs et des Bleus : au monument civique de la place publique s'oppose donc le monument religieux dans le cimetière, espace sacré mais aussi espace public. Ainsi, à Ploujean, la mémoire de la Grande Guerre a été appropriée par chaque camp : morts pour la France, la République laïque; contre martyrs chrétiens, héroïques sacrifiés pour Dieu et la Patrie.

\section{Ploujean, une commune rurale du Trégor entre châtelains et Républicains}

Cette commune du Trégor a été rattachée à Morlaix par un arrêté préfectoral du 9 février 1959. Jusqu'à cette date, elle était commune et paroisse. Elle comptait en 1911, 3568 habitants ${ }^{2}$. L'histoire de Ploujean est liée à celle de grandes personnalités. De la très longue liste de ses célébrités, on peut extraire : le corsaire Charles Cornic, les généraux Tromelin, Le Bon, et Le Flô qui fut aussi député, ministre de la guerre et ambassadeur en Russie; Édouard et Tristan Corbière, le maréchal Foch et le général Weygand et, plus près de nous, le spationaute Jean-Loup Chrétien. Ploujean possédait plusieurs atouts : sa localisation, sur la rive droite de la rivière de Morlaix, et aux portes de la dite ville, ses paysages verdoyants composés d'épaisses futaies, et d'une suite de collines, la personnalité du bourg avec son église dont la nef romane date du $\mathrm{XI}^{\mathrm{e}}$ siècle et le chœur gothique du Xve siècle.

Un autre élément fondamental est la présence considérable de châtelains. On a recensé pas moins de 17 châteaux et manoirs ${ }^{3}$. Ils étaient d'importance inégale mais leurs propriétaires, qui étaient aussi les employeurs de l'immense majorité des Ploujeannais, jouaient un rôle social et politique évident. Ils forment un bloc, clérical et monarchiste. Le général Le Flô en est un exemple typique. Propriétaire, depuis le milieu du XIX ${ }^{\mathrm{e}}$ siècle, du manoir du Nec'hoat, il " était le centre naturel et le chef du parti conservateur ${ }^{4}$ ". Au décès du général, l'hebdomadaire clérical de Morlaix La Résistance, reprit, le 26 novembre 1887, un extrait de l'oraison funèbre qui résumait la pensée politique de ce général royaliste et clérical : « Faire des Français sans Dieu! cette idée étrange lui inspirait des révoltes pleines d'indignation. [...] Pour lui, Christ et France! c'était le même cri de guerre ${ }^{5}$."

2. Arch. dép. du Finistère, Guide des archives départementales, p. 346.

3. Bulletin communal de Morlaix n ${ }^{\circ}$, spécial Ploujean, janvier-février 1987 pages 30 et 31 .

4. Jean-Yves Le Goff, Le général Adolphe Le Flô, Lesneven, musée du Léon, 1993, p. 235.

5. Ibidem. 


\section{Un maréchal de France à Ploujean}

Ploujean ne se distingue pas réellement des autres communes bretonnes pendant le premier conflit mondial. Elle a toutefois une singularité : celle de compter parmi ses administrés le général Ferdinand Foch. Celuici, originaire de Tarbes mais marié à une bretonne, Julie Bienvenüe, acheta le 16 août 1895 le manoir de Traonfeunteuniou. Il s'agissait d'un grand domaine de 66 hectares composé de plusieurs fermes, d'un manoir et de ses bâtiments de service et d'une chapelle ${ }^{6}$. Dès lors, la famille y passait les mois d'été et le général les y rejoignait dès qu'il le pouvait. Il était d'ailleurs à Ploujean lorsqu'il reçut l'ordre de rejoindre sa garnison alors que le déclenchement de la Grande Guerre était imminent.

À deux reprises durant le conflit, le conseil municipal envoya ses félicitations à Foch. "Interprétant les sentiments patriotiques de la population ", le conseil félicita " le général Foch à l'occasion de sa nomination de commandant en chef des armées alliées en France. " Puis, le 18 août 1918, la municipalité adressa " un hommage respectueux au maréchal Foch à l'occasion de son élection à la dignité de maréchal de France. " Les élus " adressent l'expression de leur gratitude au grand chef qui conduit les soldats héroïques à la lutte contre l'envahisseur pour le bien de la civilisation et de la liberté ${ }^{7}$ ". On imagine l'aura provoquée par la présence d'une telle personnalité à Ploujean. Aujourd'hui, le souvenir de Foch est toujours vif auprès des anciens qui le racontent allant à la messe le dimanche avec sa famille, s'installant dans la stalle offerte par "sa grandeur Monseigneur Duparc, évêque de Quimper et de Léon, le clergé, le conseil paroissial et les paroissiens de Ploujean ${ }^{8}$ " en 1916. Un autre souvenir reste assez vivace, il est lié aussi à sa venue à la messe dominicale, dans les mois qui suivirent la fin de la guerre, en compagnie de Pétain et de Weygand ${ }^{9}$. Foch, qui avait un frère jésuite, était profondément croyant et assistait chaque dimanche, en famille, à la messe. Son banc d'honneur était aux côtés de ceux des châtelains. Il participa également à la remise des prix de fin d'année aux filles de l'école privée, installée dans les locaux fournis par le comte de Beaufort du châteaud de Keranroux. Ces souvenirs sont assez symptomatiques car ils associent inévitablement Foch au parti de l'Église et à la Première Guerre mondiale. Cette présence renforce le poids des châtelains car ce généralissime est l'un d'eux. Mais Ploujean est une terre radicale où les deux camps s'affrontent pour s'approprier la mémoire combattante.

\section{La mémoire combattante disputée}

Edmond Rostand l'avait suggéré en 1917, dans son livre Comment glorifier les morts pour la patrie? : " le plus modeste soldat doit savoir en mou-

6. Jean Autin, Foch ou le triomphe de la volonté, Paris, Perrin, 1998, p. 70.

7. Délibérations du conseil municipal de Ploujean, 18 août 1918.

8. Cette stalle ou banc d'honneur est toujours visible dans l'église de Ploujean.

9. Entretien avec Jean Jacob, maire-adjoint de Ploujean, le 23 mai 2001. 
rant que son nom sera écrit sur un mur dans un lieu en évidence où les yeux prévenants pourront les rechercher et les yeux rêveurs s'en éclairer $^{10}$ ". La guerre a été un tel traumatisme qu'il est apparu nécessaire de communier dans la célébration des disparus. Ainsi, la loi du 25 octobre 1919, sans aucun caractère obligatoire, proposait aux communes de faire bâtir des monuments du souvenir. Comme partout en France, les autorités municipales de Ploujean souhaitèrent honorer les victimes de la Grande Guerre. D'autant plus qu'avec 142 morts pour la France, cette commune avait payé un lourd tribut à la victoire, comme l'ensemble de la Bretagne, fortement rurale. Ploujean, avec 3,97 \% de sa population tuée entre 1914/1918, serait exactement dans la moyenne bretonne si l'on tient compte des estimations actuelles des historiens ${ }^{11}$.

Comme l'a dit Michel Winock, "la culture politique française est une culture historique de guerre civile ${ }^{12} "$. Ainsi, le projet de monument aux morts peut-il symboliser à lui seul l'enjeu de mémoire entre les deux camps opposés : l'union sacrée, forgée au feu, ne résista pas au retour de la paix. À Ploujean, plus qu'ailleurs, "l'enjeu n'est pas seulement commémoratif, il est aussi politique ${ }^{13}$ ». Afin de mener à bien ce projet, le conseil municipal adopte la création d'une commission extra-municipale chargée d'organiser une souscription publique et de demander l'avis de la population ${ }^{14}$. Après une première proposition de bas-relief, plaqué sur la face Est de l'ossuaire, qui s'avéra être trop coûteuse, le maire proposa, le 10 janvier 1921, d'ériger un monument de type pyramidal. Le seul débat qui divisa alors les conseillers municipaux fut son emplacement : certains souhaitèrent qu'il soit installé au centre du bourg, bien visible de tous. Le maire refusa estimant que l'on ne "saurait songer à la place publique car il ne resterait plus alors l'espace suffisant aux besoins normaux de la commune ${ }^{15}$ ». Le culte du souvenir ne devait donc pas gêner l'activité des vivants. Ce projet, très classique, au coût de 6072,30 francs, est adopté sans aucun problème par la commission départementale le 6 avril $1921^{16}$. On le voit, l'érection de ce monument n'a pas suscité de querelles particulières. Si cela s'est déroulé ainsi, c'est parce que l'autre camp, celui des châteaux et du presbytère, avait déjà œuvré pour avoir "son " monument. Il pouvait donc se désintéresser de ce monument civique car il ne serait pas le sien.

10. SiRINELLI, Jean-François (dir.), Histoire des droites en France, t. 2, Cultures, Paris, Gallimard, 1992, p. 506.

11. Croix, Alain et VeILlaRd, Jean-Yves (dir.), Dictionnaire du patrimoine breton, Rennes, éditions Apogée, 2000, article sur la " Guerre de 1914-1918 " par Didier GuIVARC'H.

12. Winock, Michel, La France politique, $X I X{ }^{e}-X X^{e}$ siècle, Paris, Le Seuil, collection " PointsHistoire ", 1999, p. 11.

13. Dictionnaire du patrimoine breton..., op. cit., article " Monuments aux morts " par Denise Delouche et Jacqueline SAINCLIVIER.

14. Délibération du conseil municipal de Ploujean, le 28 novembre 1920.

15. Idem.

16. Arch. dép. du Finistère, $4 \mathrm{~T} 77$. 
Dès la première réunion de la commission extra-municipale, " un certain nombre de membres avaient demandé qu'une croix fût apposée sur le monument ${ }^{17}$ ». Paul Cloarec, le maire avait alors répondu fermement tout en faisant preuve d'ouverture. Il justifia son refus de faire apposer tout emblème religieux sur le monument car ceci était " contraire à la loi ${ }^{18}$ ". De plus, accéder à la demande de croix sur le monument obligeait, pour respecter la législation, à ériger le monument au cimetière or le conseil entendait l'installer dans un espace visible de tous au bourg. Dès lors, il était clair que ni le curé ni les châtelains, tels que De Beaufort qui était membre, tout comme le recteur, de cette commission, ne pourraient obtenir ce qu'ils voulaient. Le maire fit aussi preuve d'ouverture : il proposa d'accorder une partie de la souscription publique afin d'élever dans le cimetière une croix " dominant l'espace réservé aux corps ramenés des champs de bataille [ce qui ] satisferait le sentiment pieux de nombreux parents ${ }^{19}$ ".

Dès lors, le recteur et les châtelains adoptèrent une nouvelle stratégie : obtenir le plus d'argent possible de la souscription publique pour financer leur projet. Suite à cette proposition de Paul Cloarec, le curé réagit ainsi : il déclare "se désintéresser de la question à moins que le produit de la souscription ne fût réparti en deux parts égales ${ }^{20}$ ". Mais il était clair que " cette exigence nouvelle dépassant la proposition faite par le maire ${ }^{21}[\ldots]$ " avait un double but : récupérer le plus d'argent possible sur la souscription et diminuer du même coup les moyens financiers nécessaires à l'érection du monument communal. Cela n'a rien d'anecdotique. En arrière plan, il y a la volonté de s'approprier la mémoire combattante. Si le maire accepte le principe d'une croix dans le cimetière c'est parce qu'elle remplacerait l'existante, vermoulue et ancienne. D'ailleurs, ses contacts pris auprès des sculpteurs locaux lui permettent de dire que l'on peut " obtenir une croix très convenable de 5 mètres de haut pour un peu moins de 2000 francs $^{22}$ ». Pour le maire, les choses sont claires : pas d'emblème religieux sur le monument communal. Si le recteur le souhaite, il peut remplacer la vieille croix du cimetière; d'autant plus qu'elle dominera le carré destiné aux soldats. En tous les cas, il souhaite " éviter toute apparence d'opposition entre deux manifestations à la gloire et à la mémoire des morts; la croix et le monument devant rester tous deux symboles d'union sur un terrain où tous les Français devraient se retrouver ${ }^{23}$ ». Il n'est donc, en aucun cas ici, question de la création d'un second monument aux morts. Et pourtant, dans l'esprit du curé, il est clair que le refus du maire d'accepter tout emblème religieux était inconcevable. Dans une lettre adressée à son évêque, le 11 décembre 1920, il juge que cette décision du maire est "d'autorité et sectaire " et que " tout

17. Délibérations du conseil municipal de Ploujean, le 28 novembre 1921.

18. Idem.

19. Id.

20. Id.

21. Id.

22. Id.

23. Id. 
en regrettant une pareille détermination qui éparpille " le souvenir il l'a " pourtant accepté en vue de l'union sacrée ». Il déplore donc la réalisation de ces deux monuments : "L'un seulement profane, le sien [celui du maire]; le second, une croix au cimetière pour donner satisfaction aux convictions des familles chrétiennes ${ }^{24}$. " Pour le curé, préserver l'union sacrée passe par l'érection d'un monument au symbole chrétien face au monument communal. Sa conception de l'union est donc exclusive et partiale. La croix doit être majestueuse : elle aura donc un Christ de $1 \mathrm{~m}$, elle s'élèvera à $6,80 \mathrm{~m}$ et occupera un soubassement de $3 \mathrm{~m}^{2}$. Le message doit être clair : elle devra comporter quatre inscriptions, rédigées en trois langues. Voici les devises que le recteur souhaite voir gravées sur le monument :

EVIT DOUE AG AR VRO (Pour Dieu et la patrie)

A LA MEMOIRE A JAMAIS BENIE DES ENFANTS DE PLOUJEAN VICTIMES DE LA GRANDE GUERRE 1914-1918

PRO VOBIS OCCIDIMUS ORATE PRO NOBIS

(Nous sommes morts pour vous, priez pour nous)

\section{PEDIT EVIDOMP ZO MARO EVIDOC'H}

(Prions pour ceux qui sont morts pour nous)

L'attitude du curé et des châtelains consista alors à pousser l'avantage obtenu par le principe d'un financement grâce à une part de la souscription publique. Tout s'accélère : le 10 janvier 1921, le conseil municipal accepte, de façon laconique et sans entrer dans le détail, le projet de croix proposé par le curé. Le 20 février 1921, le maire est mis devant le fait accompli : le curé a effectué lui-même l'adjudication pour ce marché bien que cela soit de la compétence du maire et demande au conseil municipal de la ratifier. Le conseil « après en avoir délibéré, décide d'accepter la chose faite ${ }^{25}$ ". Le marché confié à un sculpteur morlaisien consiste en une croix avec Christ pour 6200 francs, inscriptions comprises. Il s'agit en réalité d'un véritable monument aux morts.

Si le curé a cherché un appui auprès de son évêque pour contrarier les projets du maire, celui-ci cherche à maintenir une certaine concorde au sein de sa commune. Il écrit le 18 mars 1921 au préfet du Finistère pour lui demander un avis favorable auprès de la commission départementale d'examen des projets de monuments commémoratifs car : "Ce n'est pas sans peine que j'ai réussi à assurer l'union, je vous demande donc d'approuver la solution que j'ai été obligé d'adopter après de longues et difficiles négociations ${ }^{26}$. " En vue de l'inauguration, prévue le 2 octobre 1921,

24. Archives diocésaines, correspondance entre le curé de Ploujean et l'évêque, lettre du 11 décembre 1920. En décembre, le maire a toujours comme projet de faire installer un monument commémoratif en bas-relief, ce qui déplaît fortement au recteur. Dans cette même lettre, il demande conseil à son évêque : comment empêcher le maire d'utiliser le pignon Est de l'ossuaire? La réponse est décevante pour lui car l'annotation en marge indique : " pas d'atteinte au droit de jouissance, le monument étant érigé à l'extérieur ".

25. Délibérations du conseil municipal de Ploujean, le 20 février 1920.

26. Arch. dép. du Finistère, 2 O 1381. 
et afin de "vouloir pousser jusqu'au bout un esprit de large tolérance ", le maire proposa au curé de " bénir le monument et la croix " mais celui-ci

" a répondu qu'il ne savait pas s'il pourrait le faire, qu'il demanderait à son évêque. De même, le maire a invité le recteur à assister au banquet. Le recteur a répondu qu'il aurait un prédicateur. Le maire lui a dit que le prédicateur était naturellement invité. Le recteur a répondu qu'il ne pourrait s'engager pour le moment ${ }^{27}$."

La lecture de ce compte-rendu fait apparaître que les relations entre les deux camps n'étaient ni simples ni pacifiées. La volonté d'apaisement du maire se heurte à la porte close du presbytère. Le refus d'emblèmes religieux sur le monument aux morts est une faute impardonnable pour ce prêtre qui prend ainsi sa place dans la longue lignée de ceux qui, comme lui mais au siècle précédent, ne pouvaient imaginer de césure entre spirituel et temporel.

\section{Deux monuments pour deux mémoires de la Grande Guerre?}

Ce clivage politique et religieux apparaît-il sur ces deux monuments? La typologie établie par Antoine Prost permet d'en analyser le message : à chacun sa mémoire combattante; à chacun ses symboles.

Le premier monument, officiel et communal, comporte comme dédicace : " AUX ENFANTS DE PLOUJEAN MORTS POUR LA FRANCE ». Il s'agit ici de la mère patrie, de la République qui a perdu ses fils. Il présente sur chaque face les symboles du guerrier (ancre de marine, grenade, casque) et la croix de guerre " décoration due aux morts pour la France ${ }^{28}$ ». En revanche, il ne possède pas d'emblèmes religieux ce qui en fait un monument " républicain et laïque ${ }^{29}$ ". Le choix d'un emplacement à proximité de l'église ne s'explique pas par la religion mais par la volonté d'installer ce monument au milieu du village sans gêner les activités de la commune. Sa réalisation est classique : un obélisque, ou pyramide, tronqué sur un piédestal de trois marches qui est ici un support de la gloire. Le monument est entouré d'ifs qui sont des arbres funéraires dans le monde celtique. Tous ces éléments en font un monument civique.

Face à ce monument ancré dans la tradition politique communale républicaine et anticléricale, la croix concentre elle aussi de nombreux symboles et attributs porteurs de sens. Sa présence dans le cimetière en fait un monument funéraire. Symbole le plus important : la croix et son Christ en font un monument chrétien. La liste des 142 noms, classés par année, est inscrite en lettres d'or sur les quatre faces du socle. Il faut noter que les inscriptions sont différentes de celles souhaités en janvier 1921 par le rec-

27. Délibérations du conseil municipal de Ploujean, le 18 septembre 1921.

28. Prost, Antoine, Les lieux de mémoire, Tome 1 : La République, article " Les monuments aux morts ", Paris, Quarto-Gallimard, 1997, p. 205.

29. Ibidem, p. 205. 
teur. Au lieu des quatre devises en trois langues. Sa dédicace est double. Elle entend d'abord glorifier les morts : " AUX PLOUJEANNAIS HEROS DE LA GRANDE GUERRE 1914-1918 " Elle est aussi inscrite dans l'identité bretonne : " EVIT DOUE $A G A R V R O$ " (pour Dieu et la patrie). L'ensemble de ces éléments en font, selon Antoine Prost, un monument funéraire-patriotique. Le message patriotique est fortement empreint de religion : les chrétiens, morts au champ d'honneur, sont des martyrs sacrifiés pour une cause qui les dépasse individuellement. Il est clair que ceci est « aux antipodes de l'esprit républicain pour qui l'individu est la fin ultime de la société et qui proclame un message d'émancipation individuelle et collective ${ }^{30}$ ". Mais qu'au contraire, ce sacrifice "illustre le lien entre foi et Bretagne ${ }^{31}$ ".

L'inauguration des monuments est le premier acte de cette liturgie funéraire et de cette fête civique qu'est devenue très vite, par la suite, la cérémonie du 11 novembre, d'abord jour de recueillement, puis jour férié à partir de 1922. L'accueil des invités et des personnalités, ce dimanche 2 octobre 1921, se fait à la mairie par Paul Cloarec. Puis, le cortège se forme pour se rendre au service funèbre : médaillés militaires, mutilés et anciens combattants, personnalités politiques, enfants des écoles, conseil municipal, fonctionnaires, suivis de la foule des Ploujeannais, pénètrent dans l'église où attend dans la stalle d'honneur la famille Foch. La cérémonie religieuse est faite par l'abbé Roull archiprêtre de l'église Saint-Louis à Brest.

Deuxième acte : l'inauguration du monument aux morts. Le maire développe plusieurs thèmes. Il commence bien évidemment par rendre hommage aux combattants morts au champ d'honneur mais aussi aux personnalités militaires de Ploujean : le maréchal Foch et le général Weygand tous deux absents car retenus à Paris par la remise au soldat inconnu français de la médaille du congrès américain par le général Pershing. Le maire affirme ensuite, avec force, le caractère civique et patriotique du monument en justifiant son emplacement sur la place publique. Pour lui, il s'agit d'un hommage rendu aux soldats. Ces 142 noms rassemblés sur ces tables de pierre au cœur du village ont un double intérêt : conserver le souvenir et être un exemple « où les jeunes garçons viendront puiser sans cesse l'idée du sacrifice nécessaire à la vie de la patrie, où les jeunes filles viendront chercher le courage nécessaire pour enseigner plus tard à leurs enfants la grande notion du devoir national ${ }^{32}$ ». Ces noms classés par ordre alphabétique montrent l'égalité républicaine devant la loi comme devant la mort mais aussi dans "le souvenir des générations futures ${ }^{33}$ ". Ainsi, ce monument doit-il exprimer le patriotisme et le civisme républicain à destination des enfants des écoles, garçons comme filles.

30. Ibid., p. 208.

31. Dictionnaire du patrimoine breton, op. cit., article de Didier GUYVARC'H sur la " guerre de 1914-1918".

32. L'Éclaireur du Finistère, samedi 8 octobre 1921.

33. Prost, Antoine, Les lieux de mémoire, op. cit., p. 209. 
Le troisième acte est l'inauguration de la croix dans le cimetière. Devant le monument aux morts, Paul Cloarec avait expliqué la position du conseil municipal par rapport à la croix. Il avait décrit un cortège de veuves venues lui dire : "Après avoir glorifié nos morts, nous voudrions pleurer à l'ombre du symbole qui nous apparaît comme le gage d'une espérance pour la vie future ${ }^{34}$." Si le conseil municipal a accepté c'est pour respecter toutes les croyances et parce que ses convictions républicaines lui permettaient de considérer que " nos morts sont si grands que chacun peut, sans crainte, leur sacrifier un peu de ses préférences; ils n'avaient pas tous la même philosophie, tous ont eu le même courage et ont été également frappés par les obus ou les torpilles ennemies sous le même uniforme $^{35}$ ". La position du maire est claire : si le monument aux morts est à la gloire des combattants; la croix " sera la tombe où l'on pleure et où l'on prie $^{36}$ ". Le recteur a une vision exclusive de la mémoire combattante : les poilus sont les " martyrs du patriotisme " auxquels on devait élever " ce calvaire, témoignage de foi et de reconnaissance à l'égard de ces disparus $^{37}$ ". Ce monument est le seul qui compte; l'autre, le profane n'existe pas. Pour lui, ces sacrifiés sont issus d'une " race de géants " et sont des " héros devant qui pâtissent les gloires de l'antiquité ${ }^{38}$ ". Le curé n'évoque pas les problèmes liés à l'érection de la croix et entend revenir sur le thème de l'union sacrée :

«Mais surtout soyons unis. Plus de querelles intestines, plus de luttes fratricides, mais la paix et la concorde dans le respect mutuel de toutes les saines libertés. [...] Et travaillons. Laboremus selon le mot de Jeanne d'Arc, pour que la France soit toujours la patrie forte, belle et prospère, idéale, la reine des nations. Vive la France ${ }^{39}$ !»

Un calvaire dédié aux héros, une France placée sous la protection exemplaire de sainte Jeanne d'Arc : nous sommes bien face à un monument religieux, funéraire et patriotique. Dans ces discours, on tait ce qui oppose et ce qui divise, on met en valeur la concorde nationale. Mais au plan communal, il y a les faits. Et les "faits sont têtus " : avec deux monuments commémoratifs peut-on encore parler d'union sacrée?

À Ploujean, il y a donc deux monuments commémoratifs de la Grande Guerre. Mais cette originalité se renforce encore par la tenue de deux cérémonies bien distinctes. Chaque camp a son rituel : républicain et laïque d'un côté; religieux de l'autre. C'est une vraie opposition qui s'affirme, alors que s'est élaborée " pour honorer la génération perdue, [...] un ensemble tragique : unité de temps, le 11 novembre; unité de lieu, le monument aux morts; unité d'action, la cérémonie commémorative ${ }^{40}$ ». On le voit ici deux éléments

34. L'Éclaireur du Finistère, samedi 8 octobre 1921.

35. Ibidem.

36. Ibid.

37. Ibid.

38. Ibid.

39. Ibid.

40. BECKER, Annette, «Les monuments aux morts », L'Histoire, n 225, octobre 1998, p. 51. 
de cette trilogie n'existent pas : ni l'unité de lieu, ni l'unité d'action. La mémoire de ces doubles commémorations reste vivace chez certains anciens qui racontent encore aujourd'hui avec précision le rituel ${ }^{41}$. Cela commençait par l'affirmation d'un clivage spirituel : la messe du souvenir à laquelle refusaient de participer certains élus radicaux et les laïcs. Puis, le cortège sortait de l'église et se scindait immédiatement : la foule laïque, les autorités municipales et les anciens combattants se rendaient devant le monument aux morts. Là, se déroulait la cérémonie de recueillement et l'appel aux morts auquel participaient, avec leur instituteur, les enfants des écoles publiques. Tandis que, dans le même temps, mais au cimetière, se tenait la cérémonie religieuse et concurrente menée par le curé et à laquelle assistaient l'ensemble des châtelains avec leurs familles de métayers et d'ouvriers agricoles dont la plupart des enfants étaient scolarisés à l'école privée.

Les familles des châtelains les plus importants de Ploujean figurent parmi les victimes de la Grande Guerre. Deux fils du comte Gouyon de Beaufort, du château de Keranroux, furent tués, l'un en 1914; l'autre en 1916. L'église paroissiale possède d'ailleurs, la bannière de Keranroux représentant saint François-Xavier et saint Jean-Baptiste; bannière réalisée avec le tissu de leurs tenues militaires. Mais c'est sans doute la disparition, à Dixmude, du vicomte Alfred de Nanteuil de la Barre du château du Nec'hoat, qui frappa durablement les esprits. Il fut tué au combat le 10 novembre 1914. La semaine religieuse dressa un portait élogieux de ce lieutenant de vaisseau frappé " en chargeant l'ennemi à la tête de ses marins ${ }^{42}$ ». Il s'agissait du petit-fils d'Adolphe Le Flô pour qui « la cause de la religion [...] s'identifiait avec celle de la France ${ }^{43}$ ". D'autant plus qu'il n'avait jamais caché ses sentiments monarchistes car, il considérait, comme son grand-père, que " la monarchie était l'outil nécessaire au relèvement national et religieux ${ }^{44}$ ". Pour ces familles, Gouyon de Beaufort et Nanteuil de la Barre, la gloire de leurs héroïques soldats et le souvenir, empreint d'espérance de résurrection, ne pouvaient s'incarner que dans ce calvaire mêlant religion chrétienne et patriotisme. Et certainement pas dans ce monument aux morts civique qui, comme bien d'autres en France, est marqué par "son dépouillement un peu froid, sa laïcité un peu sèche ${ }^{45}$ ".

Le thème de l'union sacrée évoqué tour à tour par le maire et le curé lors de l'inauguration de 1921 se heurte à la réalité et surtout au poids du passé politique et religieux de Ploujean. Car pour comprendre l'existence d'un tel clivage il est nécessaire de chercher des réponses dans le long terme et d'aller voir comment furent vécues les grandes questions qui divisèrent la France du début du $\mathrm{xx}^{\mathrm{e}}$ siècle : la fin des congrégations et la séparation de l'Église et de l'État avec son cortège d'inventaires.

41. Entretien avec Jean Jacob, le 23 mai 2001.

42. La semaine religieuse, année 1913, p. 796.

43. Ibidem, p. 797.

44. Ibid.

45. Prost, Antoine, Les lieux de mémoire, op. cit, p. 209. 


\section{Ploujean et les grandes lois du début $\mathrm{du}_{\mathrm{Xx}}^{\mathrm{e}}$ siècle : à l'origine de la fracture?}

Les grandes lois républicaines du début du siècle trouvèrent à Ploujean, comme ailleurs, un écho très important; à la mesure de la fracture qui augmente alors entre les partisans de la république et le monde des châteaux et du presbytère. Ce fossé est d'autant plus large que le maire de Ploujean, Émile Cloarec, est alors une grande personnalité républicaine.

L'expulsion des congrégations sonna comme le premier acte permettant d'illustrer les deux camps en action. Les petites sœurs du Saint-Esprit enseignaient dans un bâtiment mis à disposition par le châtelain de Keranroux, le comte de Beaufort. Or, en application de la loi sur les congrégations, leur expulsion fut ordonnée : le commissaire de police de Morlaix, accompagné de deux brigades de gendarmerie et d'une compagnie d'infanterie, se posta donc devant la porte de l'école des sœurs le 12 août 1902 à 8 heures 30 .

Son rapport au préfet du Finistère est explicite :

"Arrivé devant la porte et après les sommations faites, Monsieur le comte de Beaufort, propriétaire de l'immeuble, m'ayant répondu que l'on n'ouvrirait pas, j'ai ordonné à un serrurier de la localité, requis par Monsieur Cloarec, d'enfoncer la porte. Cette opération terminée, je suis entré dans la cour et je me suis trouvé en présence de Messieurs de Beaufort, de Nanteuil, d'Honinctun, Dulong de Rosnay. [...] Des cris sont poussés de "vivent les sœurs, vive la liberté." J'arrive devant la porte intérieure de l'établissement. Elle est fermée. Je la fais enfoncer et je me trouve en présence de cinq rangées de chaises, sur les quatre premières se trouvent les familles des manifestants. Je les fais sortir les unes après les autres. La résistance est toute passive. L'expulsion se produit pour les sœurs et je procède à l'apposition des scellés. Il n'y a eu aucune violence d'exercée, quelques cris ont été poussés de "À bas Cloarec, À bas Combes" mais dans la foule il m'a été impossible de déterminer les personnes qui en étaient l'auteur ${ }^{46}$."

Dans une fiche adressée au préfet, le commissaire de police précise que le recteur qui était présent lors de ces événements, avait laissé sonner le tocsin à l'église et que surtout : " il a abandonné un service funèbre commencé pour se rendre à la manifestation ${ }^{47}$ ". En réalité, cette agitation ne doit pas laisser penser que la population soutenait les petites sœurs. Les gendarmes, dans leur procès-verbal de renseignement, du 24 juillet 1902 , estimaient que " la population était en grande majorité indifférente mais environ 300 personnes en tête desquelles se trouvent messieurs de Beaufort, de Nanteuil, Cazin d'Honinctun, et Dulong Rosnay protestent ${ }^{48}$ ". La description du camp clérical est claire : les châtelains, leur famille et

46. Arch. dép. du Finistère, 1 V 1156. Dans l'édition du 14 août 1902, l'hebdomadaire catholique La Résistance, précisait que les châtelaines aussi étaient présentes : $\mathrm{M}^{\mathrm{me}}$ la comtesse de Nanteuil, fille du général Le Flô, $\mathbf{M}^{\mathrm{me}}$ d'Honinctun, $\mathbf{M}^{\mathrm{me}}$ de la Vallière, $\mathbf{M}^{\mathrm{me}} \mathrm{de}$ Lugré, $\mathrm{M}^{\mathrm{me}}$ de la Briffe, $\mathrm{M}^{\mathrm{me}}$ de Saint-Laon.

47. Ibidem.

48. Ibid. 
leur personnel; le curé et les religieuses. De l'autre, le maire Émile Cloarec et la grande majorité de la population aux sentiments républicains.

L'homme qui incarna cette politique combiste est Émile Cloarec (18581914). Licencié en droit, il fut successivement avocat, avoué puis juge suppléant au tribunal civil de Morlaix. Il mena une carrière politique très active : maire de Ploujean de 1892 à 1914; député de Morlaix en 1901, il fut réélu en 1902, 1906, 1910, 1914. Il fut également conseiller général du canton de Saint-Thégonnec entre 1910 et 1914. Pour ce modéré, élu au centre gauche et siégeant au sein de la Gauche démocratique puis des Républicains de gauche, la question religieuse et au cœur de son combat. Émile Cloarec, élu grâce aux radicaux et aux ouvriers morlaisiens, précise dans un tract électoral qu'il se veut " ni trop à gauche, ni trop à droite ". En revanche, il affirme un engagement très fort en faveur de la politique de laïcisation et fut intransigeant sur la question religieuse dans cette circonscription de Morlaix, venue à la République par l'anticléricalisme. Dans un autre tract, rédigé en breton et en français, il s'explique, en 1906, sur son vote en faveur de la séparation de l'Église et de l'État : " [...] ne pouvant admettre que le pape se crut le droit de donner des ordres au Président de la République, j'ai voté la séparation de l'Église et de l'État... J'ai considéré que la noblesse et le clergé devait obéissance aux lois comme tous les autres citoyens français, ce qu'ils ne pouvaient pas admettre... " Émile Cloarec représenta tout ce que détestaient les cléricaux et les châtelains. Ses votes à la Chambre des députés sont sans ambiguïtés : le 24 mars 1904, il vote pour « l'ensemble du projet de loi relatif à la suppression de l'enseignement congréganiste "; le 14 janvier 1905, il vote en faveur du gouvernement Combes; le 3 juillet 1905, il vote en faveur de la séparation des Églises et de l'État et le 13 novembre 1906, il vote contre l'application de cette loi de séparation " avec tolérance ${ }^{49}$ ". Au plan local, c'est lui qui fit construire, en 1905, la mairie et l'école primaire. D'ailleurs, il introduit, dans la maison commune, le buste de Marianne ce qui à l'époque " est un choix militant ${ }^{50}$ ". Ce buste de la République comporte les attributs de " Marianne au combat " : le bonnet phrygien, la cocarde tricolore, le lion, symbole de la force populaire, sur la poitrine. L'ère Cloarec est républicaine.

Ce notable de gauche déplaît à ceux de droite. Ses votes, à la Chambre des députés, sont des déclarations de guerre sociale et religieuse; d'autant plus qu'ici « le presbytère est presque toujours l'écho du château ${ }^{51}$ ". Cette hostilité est visible en particulier dans la presse catholique qui prend de manière systématique Émile Cloarec pour cible. S'il est qualifié le 13 septembre 1902 de "robespierrot ${ }^{52}$ ", les attaques peuvent se révéler

49. Informations extraites de PASCAL, Jean, Les députés bretons de 1789 à 1983, Paris, PUF, 1983.

50. Agulhon, Maurice et Bonte, Pierre, Marianne, les visages de la République, Paris, Gallimard, 1992, p. 45.

51. Croix, Alain et Roudaut, Fañch, Les Bretons la mort et Dieu de 1600 à nos jours, Paris, Messidor/Temps actuels, 1981, p. 197.

52. La Résistance, le 13 septembre 1902. 
beaucoup plus cruelles. Un exemple, au goût douteux, peut illustrer cette haine. Sous le titre "Cérémonie funèbre à Ploujean ", voilà ce que les lecteurs de La Résistance purent lire :

" [...] Dimanche dernier, les conseillers municipaux et un grand nombre d'habitants de la commune avaient été conviés à un grand repas funèbre, en mémoire de M. Cloarec. Pour rappeler le sombre fleuve du Cocyte, le long duquel errent sans trêve les mânes plaintifs; c'est sur les bords du Dourdu (l'eau noire) que se sont tenues ces agapes funéraires. En Trégor, comme en Cornouaille, et en Léon, le culte des Trépassés est toujours vivace, les rancunes s'apaisent et les haines s'éteignent en face de la mort. Aussi, beaucoup ont tenu à prendre leur part des obsèques du député-maire, qui sans doute en d'autres occasions, se seraient abstenus de répondre à l'invitation lancée. [...] L'émotion n'a plus connu de bornes, lorsqu'un des orateurs après avoir rappelé ce qu'eut pu être au parlement le député de Morlaix, si malheureusement sacrifié à la loi d'association, s'écrie sur un ton lamentable : Adieu M. Cloarec, Adieu. Signé : un Ploujeannais ${ }^{53}$."

C'est bien sûr la bataille autour des Inventaires qui accentua le fossé entre ces deux camps. Pour les châtelains et le curé, le vote de la séparation de l'Église et de l'État est une faute impardonnable ${ }^{54}$.

Le second grand moment d'opposition entre laïcs et cléricaux fut la réalisation des Inventaires en 1906. L'intervention du pape Pie X, qui condamna la Séparation dans son encyclique Vehementer nos, encouragea les fidèles à résister. À Ploujean, on retrouve l'alliance du presbytère et du château, union inquiétante pour le sous-préfet de Morlaix qui estime qu'il ne pourra faire l'inventaire " qu'avec force armée " car le curé a déclaré " qu'il ne cédera qu'à la force pour donner satisfaction aux désirs du pape et de l'évêque de Quimper ${ }^{55}$ ". Le sous-inspecteur de l'Enregistrement chargé de l'Inventaire se présenta le 26 février 1906 au presbytère mais fut "laissé à la porte sous la pluie pendant 31 minutes $^{56}$ ". Émile Cloarec entre alors en scène. Il écrit depuis Paris au recteur de Ploujean pour l'aviser que l'inventaire aura lieu le vendredi 2 mars à 14 heures. Sa lettre mêle flatterie et menace : "Ayant eu l'occasion d'apprécier votre courtoisie et votre intelligence, je suis persuadé que cette opération, qui n'a aucun caractère vexatoire, aura lieu sans troubles et sans manifestations, mon devoir est de me rendre à Ploujean pour le maintien de l'ordre et le respect des lois. " Puis, Émile Cloarec demande au recteur s'il doit envisager de rentrer ou si cela se passera dans le calme. La réponse du curé, fortement teinté d'ironie, est révélatrice des rapports entretenus entre la mairie et le presbytère :

53. Ibidem, le 30 août 1902.

54. Voici une anecdote assez révélatrice. Lors de l'inauguration, en 1980, de la nouvelle école publique dénommée Émile Cloarec, il y eu un petit incident : les descendants de Cloarec furent présentés à ceux d'un châtelain de Ploujean. Ceux-ci redirent avec force combien ce vote avait été, et était toujours à leurs yeux, une faute inqualifiable. Entretien avec Jean Jacob le 23 mai 2001.

55. Arch. dép. du Finistère, 2 V 41, Télégramme codé, sans date, du sous-préfet de Morlaix au préfet du Finistère.

56. Idem. 
"Monsieur le député, votre présence à Ploujean, à pareille occasion, serait une cause de troubles. Je crois pouvoir répondre du calme de ma population; par conséquent ne vous dérangez pas, uniquement pour cela. Votre dévoué Grall, recteur de Ploujean. " Mais comme le redoutait le députémaire, son " dévoué " recteur fit de la résistance. Le 2 mars, le même fonctionnaire se représente mais se voit refuser l'entrée de l'église par le recteur et par le comte de la Barre de Nanteuil. L'Inventaire fut repoussé au lendemain. Les autorités demandèrent alors la protection de la troupe et de la gendarmerie : le 3 mars à 6 heures du matin, l'église est cernée. La serrure est brisée, après les sommations, par un soldat armé « d'un pic de campagne $^{57}$ ". L'Inventaire est effectué pendant que les fidèles prient et chantent. Avant de laisser pénétrer le sous-inspecteur de l'Enregistrement dans l'église, le recteur et le comte de la Barre de Nanteuil lui ont remis deux lettres de protestation. Le curé se place dans la ligne définie par Pie X:

"Ce que le pasteur infaillible a flétri et condamné, je ne peux l'approuver. Préposé à la garde de cette église, je me fais un devoir des plus sacrés de défendre, de toute ma puissance, ses droits et ses intérêts. L'œuvre que vous êtes venus accomplir aujourd'hui est considérée par la France catholique tout entière comme les préliminaires de la spoliation de nos églises. Je proteste de toutes mes forces contre cet acte odieux et je vous déclare que je ne prêterai aucun concours à l'inventaire que vous êtes venus commencer. Yves Grall, recteur de Ploujean ${ }^{58}$."

Le président du bureau des marguilliers de la fabrique de Ploujean est le comte de la Barre de Nanteuil. Cet influent châtelain s'oppose, au nom de la fabrique, à l'Inventaire en soulignant que :

"Les inventaires ne se font qu'après décès, or la fabrique de Ploujean, personne morale, n'est pas morte. Elle est vivante et bien vivante, et j'ose le dire, elle ne périra pas, car des jours meilleurs luiront peut-être bientôt pour le pays. [...] Notre résistance est donc justifiée. Elle est absolument légitime et conforme au droit naturel $[\ldots]^{59}$."

Il est clair que ces événements creusent encore davantage le fossé entre les deux France. Le représentant à Morlaix de cette politique de laïcisation n'est autre que le député-maire de Ploujean. Il est alors de plus en plus brocardé par la presse catholique qui le surnomme «le plus grand crocheteur du Trégor " et pour qui il "n'est qu'un député blocard ${ }^{60}$ ". Cloarec resta dès lors la cible de la droite cléricale. Des années plus tard, lors de la campagne législative de 1914, une chanson en breton, diffusée sur feuille volante, l'attaqua directement. Son titre était sans ambiguïté " Pilomp Émile " c'està-dire : "Battons Émile. " Il y est associé à "Caillaux-Rochette, les filous ". Et pire, on l'accuse de ne pas fréquenter l'église car : "Avec les Francs-

57. La Résistance, 10 mars 1906.

58. Arch. dép. du Finistère, 2 V 41. Lettre du 2 mars 1906 du recteur de Ploujean à

l'Inspecteur de l'Enregistrement.

59. Idem.

60. La Résistance, le 10 mars 1906. 
Maçons, il va rue de Valois à Paris, comme cela vous ne le prendrez pas pour un blanc ${ }^{61}$ !"

\section{À Ploujean, l'opposition entre mémoires laïques et cléricales est liée à l'histoire}

Au terme de cette étude, une conclusion s'impose : la mémoire disputée de la Première Guerre mondiale trouve son origine, à Ploujean, dans les grandes batailles de la République et la politique de laïcisation du début du $x^{e}$ siècle. Les deux camps qui se font face sont les mêmes. Malgré le temps qui passe et les changements de maires et de curés, la mémoire de ces fractures et l'influence sociale, toujours prédominante, des châtelains sont décisives.

Pour la droite cléricale et monarchiste, il faut faire bloc autour de la religion et du curé. Ici, les châtelains jouent le rôle majeur : ils président la fabrique de l'église, ils fournissent les immeubles nécessaires aux congrégations jusqu'en 1902, puis mettent une maison à disposition de l'association s'occupant de l'école privée catholique. Autre élément, qui derrière l'anecdote montre cette union du château et du presbytère : les cloches de l'église datant de 1870 ont Caroline de la Barre de Nanteuil, fille Le Flô, et Charles Tixier Damas de Saint Prix pour marraine et parrain. En 1936, la nouvelle cloche voit la tradition respectée avec la maréchale Foch pour marraine et le vicomte du Halgoët pour parrain. Pendant la Grande Guerre, le prêtre de Ploujean écrit à son vicaire général qu'il a réussi à avoir au catéchisme les enfants pauvres mais surtout : "Les enfants des familles très riches : j'ai réussi et ces personnes riches m'en savent gré maintenant : elles viennent m'aider pour le catéchisme, elles ou leurs préceptrices; elles connaissent dans quelle ignorance crasse sont laissés certains enfants ${ }^{62}$." La position intransigeante du curé concernant le monument aux morts s'explique mieux ainsi. Compte tenu de ce passé, ni lui, ni les châtelains, ne pouvaient envisager d'accepter le monument civique comme lieu de recueillement et de souvenir. Il ne pouvait être le leur car ils étaient étrangers à l'idée même de laïcité. Cette France qui sépare le spirituel du temporel n'est pas la leur. Seule une croix, honorant " Dieu et la patrie ", pouvait satisfaire leurs options politiques et religieuses. Et en faisant figurer sur son socle l'ensemble des noms des soldats tués à la guerre, n'ont-ils pas essayé d'accaparer la mémoire combattante?

Le Trégor est une terre laïque et républicaine. Ici, on n'élude pas le conflit s'il est juste et les grandes lois républicaines sont jugées émancipatrices. L'école publique et la mairie, avec son buste de "Marianne au combat ", entendent s'affirmer face à l'église. La figure républicaine d'Émile Cloarec a marqué durablement les esprits. Il fut le grand maire de Ploujean.

61. Chanson sur feuille volante. La rue de Valois était le siège du parti radical.

62. Archives diocésaines, lettre du recteur de Ploujean au vicaire général. Sans date mais rédigée pendant la guerre de 1914-1918. 
C'est son frère, Paul, qui décida de l'érection du monument aux morts sur la place du village. C'est lui qui, face à un cléricalisme fermé, proposa " l'ouverture " : il accepta le principe de la croix dans le cimetière - mais avaitil mesuré qu'elle deviendrait ce contre-monument? - et invita pour l'inauguration de 1921 trois députés représentant les trois listes en présence aux législatives de 1919 : Émile Goude pour les socialistes, Georges Le Bail pour les radicaux et Vincent Inizan pour les conservateurs ${ }^{63}$. Là encore, il manifestait un républicanisme de conviction mais ouvert. Malgré cette bonne volonté, son souci d'éviter la concurrence entre les deux monuments n'a pas porté : la mémoire de la Grande Guerre à Ploujean est restée double.

Quel était l'avenir de ces deux mémoires combattantes? Les deux cérémonies se déroulèrent jusqu'au milieu des années trente. Le fait majeur fut l'accord passé en 1935 par un ancien maire radical, Eléouet, avec la droite ploujeannaise. Cette alliance de circonstance obligea les conservateurs élus à assister à la cérémonie du 11 novembre au monument aux morts ; même si ensuite ils pouvaient aller devant la croix du cimetière. En obtenant ces responsabilités municipales, la droite s'était républicanisée. D'ailleurs, la date qui peut marquer la fin de cette guerre des mémoires est 1945 : une plaque est ajoutée au monument officiel; mais pas sur la croix.

Au-delà de cet exemple, y a-t-il un « modèle Breton de sortie de l'union sacrée ${ }^{64}$ " ? Antoine Prost estime que les monuments aux morts prolongent la communion nationale ${ }^{65}$. La question peut être posée pour la Bretagne car c'est justement autour du symbole du consensus, le monument aux morts, qu'une guerre de la mémoire éclate. La trêve civile ne résista pas au retour à la paix et à la résurgence des clivages de longue durée qui rapidement l'emportèrent sur l'union sacrée. Le monument aux morts apparaît donc aussi comme un témoin de l'histoire politique et de l'histoire des mentalités; ceci d'autant plus qu'à travers lui, "les morts de la Grande Guerre entretiennent avec les survivants un dialogue permanent ${ }^{66}$ ".

63. Délibération du conseil municipal de Ploujean, le 18 septembre 1921.

64. Entretien avec Didier GUYVARC'H, maître de conférences à l'IUFM de Rennes, que je tiens à remercier tout particulièrement pour ses précieux conseils lors de la relecture de cet article.

65. Prost, Antoine Les Anciens combattants et la Société française, PFNSP, Paris, 1977.

66. BeCKER, Jean-Jacques et BERSTEIN, Serge, Victoire et frustrations, 1914-1929, Paris, Le Seuil, « Nouvelle Histoire de la France contemporaine ", n 12, 1990, p. 171. 


\section{RESUME}

Alors que généralement, les monuments aux morts de la Première Guerre mondiale prolongent l'union sacrée, c'est justement autour de ce symbole du consensus qu'une guerre de la mémoire éclate à Ploujean (Finistère). Châtelains et républicains rejouent la fracture des grandes lois du début du $\mathrm{xx}^{\mathrm{e}}$ siècle. Les deux camps sont à nouveau face à face; chacun entend s'approprier la mémoire combattante afin de lui donner sa signification : martyrs chrétiens d'un côté contre morts pour la République laïque de l'autre. Cette opposition s'affirme par l'érection de deux monuments aux morts : l'un civique et laïque, l'autre religieux. Et par la tenue de deux cérémonies commémoratives distinctes et concurrentes. Les monuments aux morts de Ploujean apparaissent donc aussi comme des témoins de l'histoire politique et de l'histoire des mentalités.

\section{ABSTRACT}

Usually the memorials to the dead of the first world war extend the united front, yet it is precisely about this symbol of the consensus that a fight of the memory broke up in Ploujean (Finistère). The nobility as well as the republicans perform again the fracture of the great laws of the beginning of the twentieth century. The two sides are facing each other again, each side wants to take over the fighting memory so as to give it its meaning : the christian martyrs on one side against the victims for civilian republic on the other. This opposition is affirmed by the construction of two monuments to the dead, one civic, the other religious. And the good behaviour of the two distinct and rival commemorations, the monument to the dead of Ploujean, also appear as evidence of the political history and of the history of the mentalitics. 
